\title{
Diseño de tareas telecolaborativas para el aprendizaje de idiomas con cuentos del mundo
}

\section{Designing telecollaborative tasks for languages learning through world tales}

\author{
Ana Sevilla Pavón \\ Universitat de València, UV (España)
}

\section{Resumen}

Este artículo se centra en el diseño de tareas de telecolaboración para el desarrollo de competencias lingüísticas, literarias, interculturales y digitales, teniendo en cuenta las diferentes acciones llevadas a cabo antes y durante el proyecto de cara a garantizar el éxito del mismo. La propuesta, realizada en el marco del proyecto iTECLA, conjuga la telecolaboración con la redacción e intercambio de cuentos que reflejan valores culturales de los respectivos países de origen de los participantes. El proyecto implica la realización de una serie de tareas por parte de cada estudiante, mediante un proceso guiado de análisis y reflexión basado en métodos de trabajo telecolaborativo en línea en contextos de aprendizaje de idiomas. Para comprobar la idoneidad de las tareas propuestas en cuanto al desarrollo de las diferentes competencias, de acuerdo con las percepciones de los propios participantes, se llevó a cabo un estudio piloto con 10 estudiantes procedentes de 3 países que completaron un cuestionario que incluía preguntas tanto de tipo abierto como cerrado. Los resultados de dicho estudio de implementación de las tareas mostraron que el 90\% de los participantes consideraban que el proyecto había cumplido completamente sus expectativas. Asimismo, los participantes otorgaron las puntuaciones máximas en relación a sus percepciones del desarrollo de la creatividad, valorando también positivamente la contribución del proyecto al desarrollo de competencias literarias, lingüísticas, interculturales y digitales. Dichos resultados apuntan a la consecución de los objetivos propuestos y ponen de manifiesto el gran potencial pedagógico de este tipo de tareas.

Palabras clave: intercambio cultural; aprendizaje de lenguas; educación inter-cultural; narración de historias.

\section{Abstract}

This article focuses on telecollaboration task design for the development of linguistic, literary, intercultural and digital competences, bearing in mind the different actions taken before and during the project so as to guarantee its success. The proposal, which stems from the iTECLA project, joins telecollaboration with the writing and sharing of stories which reflect cultural values from the participants' respective countries of origin. The project involves the 
completion of a set of tasks by students in a guided process of analysis and reflection based on methods of online telecollaborative work in languages learning contexts. In order to test the appropriateness of the proposed tasks with regard to the development of different competences, according to the perceptions of the students themselves, a pilot study was carried out with 10 participants from 3 different countries. They completed a questionnaire with both open-ended and closed questions. The results of this study about task implementation showed that $90 \%$ of the participants felt that the project had completely fulfilled their expectations. Furthermore, participants gave the highest scores in those questions related to their perceptions about the development of creativity, while also rating very positively the project's contribution to the development of literary, linguistic, intercultural and digital competences. These results indicate the achievement of the goals while revealing the great pedagogical potential of these kinds of tasks.

Keywords: cultural exchange; languages learning; intercultural education; story telling.

El siglo XXI plantea numerosos desafíos a nivel educativo en todo el mundo que solo pueden enfrentarse con un cambio de paradigma, pasando de la visión decimonónica del proceso de enseñanza-aprendizaje a una visión más holística y centrada en el alumno, flexible, creativa, compleja, basada en tareas (Ellis, 2000; Willis y Willis, 2007) y que permita que se creen nexos de unión entre el aula y el mundo exterior (Nunan, 1991; Tomlinson, 2011). Del mismo modo, en la educación superior se plantea la necesidad de colaborar con naciones y entre naciones, de que haya un mayor entendimiento entre culturas y de fomentar la internacionalización (Teichler, 2004; Altbach y Knight, 2007; Sevilla-Pavón, 2015). Vivimos en tiempos de cambios rápidos en la organización social, las relaciones interpersonales y el modo en que el conocimiento se transmite y gestiona, hasta tal punto que no se trata simplemente de una época de cambios, sino más bien de un cambio de época (Castells, 2001). Así, emerge un nuevo modelo de sociedad caracterizada, además de por el fácil y rápido acceso y transmisión de información y conocimiento, por el hecho de que la producción, generación y procesamiento del conocimiento y de la información están mediados por nuevos dispositivos tecnológicos. Dichos dispositivos permiten la difusión y procesamiento en tiempo real y a escala mundial en el conjunto de los procesos que constituyen la actividad humana (Castells, 2001).

En este sentido, el mayor reto del sistema educativo actual, de acuerdo con Vinagre Laranjeira (2010a), tiene que ver con la formación de profesionales capacitados para incorporarse y participar plenamente en una sociedad en que el conocimiento es el recurso fundamental para el desarrollo socioeconómico. Para la formación de los futuros profesionales es necesario, a su vez, el surgimiento de nuevas prácticas educativas que tengan en cuenta el papel fundamental que las Tecnologías de la Información y de la Comunicación (TIC) desempeñan en el modo en que se accede y comparte la información y el conocimiento, lo cual requiere el desarrollo de una serie de competencias que permitan a los futuros profesionales afrontar esta nueva 
realidad con éxito, siendo capaces de aprender a aprender (Gee, 2007), al tiempo que construyen una nueva mente mediada por instrumentos tecnológicos con ayuda de "prótesis cognitivas" que, finalmente, sean incorporadas e incluso naturalizadas por sus usuarios (Monereo y Pozo, 2008). Del mismo modo, en dichos procesos de aprendizaje se ha de tener en cuenta el hecho de que, hoy en día, las TIC se encuentran en el centro de la comunicación y provocan importantes modificaciones de nuestras habilidades cognitivas, como ya lo hizo en el pasado la transmisión oral del conocimiento y de la información. De este modo, se modifican las formas de aprender y, consecuentemente, habrían de cambiar también las formas de enseñar (Chapelle y Hegelheimer, 2004; Monereo y Pozo, 2008; Compton, 2009).

Ya en 1998, el impacto de las TIC y las exigencias de la nueva Sociedad de la información y el conocimiento fueron el tema principal del Informe mundial de educación de la Organización de las Naciones Unidas para la educación, la ciencia y la cultura (UNESCO): Los docentes y la enseñanza en un mundo en mutación (UNESCO, 1998). Dicha publicación describía las innovaciones y los cambios provocados por las TIC en las metodologías de enseñanza, las cuales, a su vez, han venido motivando una profunda transformación en el proceso de enseñanzaaprendizaje en varios niveles: objetivos, contenido, metodología, y evaluación y papel de alumnos y profesores, tanto en el aula como fuera de ella (Vinagre Laranjeira, 2010a). Diez años después, en 2008, la UNESCO se refirió nuevamente al papel de las TIC en "Estándares de competencia en TIC para docentes" (UNESCO, 2008), afirmando que para vivir, aprender y trabajar de manera exitosa en un mundo y en un mercado cada vez más complejos, ricos en información y en conocimiento, tanto estudiantes como profesores deben ser capaces de usar la tecnología digital con eficacia (UNESCO, 2008).

Además de los desafíos tecnológicos, el siglo XXI también ha venido acompañado de retos derivados del creciente nivel de globalización y de interconexión de los ciudadanos del planeta. Se han alcanzado cifras sin precedentes de movimientos migratorios a escala mundial de muy variados grupos sociales, entre los que se incluyen estudiantes, trabajadores y refugiados (Piqueras Haba, 2011). Estos procesos exponen tanto a los individuos migrantes como a las sociedades de acogida a nuevos códigos lingüísticos y culturales.

La necesidad de saber desenvolverse con éxito en estos entornos plurilingües y multiculturales contrasta con la realidad de muchos alumnos, cuya situación socioeconómica o familiar, en ocasiones, dificulta que puedan tener una experiencia de estudio o trabajo en el exterior (Comisión Europea, 2013). Ello les sitúa en desventaja frente a aquellos que sí pueden beneficiarse de ese tipo de intercambios, ya que las experiencias internacionales dotan a los participantes de competencias que les capacitan para enfrentarse a un mundo cada vez más globalizado e interconectado. Así, el entorno empresarial afirma que los candidatos con experiencias internacionales previas tienen una mayor probabilidad de adquirir competencias clave que favorecen su capacidad de inserción laboral (Lichy y 
Favre, 2018), tales como: flexibilidad, autonomía, liderazgo, innovación, madurez, creatividad, ambición, capacidad para realizar presentaciones, independencia y preocupación por otras culturas. Sin embargo, como se ha señalado anteriormente, existe una evidente carencia de experiencia internacional entre los universitarios de España y Europa ya que, a pesar de que el objetivo de la Unión Europea es que, en 2020, al menos el $20 \%$ de los estudiantes realice parte o la totalidad de sus estudios o prácticas en un país extranjero, en la actualidad tan solo el 10\% lo hace, ya sea con apoyo de la beca Erasmus (4,5\%) o de otras entidades públicas o privadas (Comisión Europea, 2013). La pregunta que surge a partir de estos datos es: ¿qué se puede hacer para que el $90 \%$ restante de los estudiantes que no pueden irse fuera debido a su situación socioeconómica tenga la posibilidad de aprender idiomas y de tener una experiencia intercultural parecida a la que vivirían en un contexto de inmersión, pero sin moverse de casa? Una posible respuesta es ofrecer un marco adecuado para la puesta en práctica de tareas de telecolaboración o de intercambio intercultural en línea que permitan el desarrollo profesional de los estudiantes, fomentando competencias digitales, interculturales, literarias y lingüísticas a través de una adecuada integración de prácticas telecolaborativas en la formación lingüística de la población universitaria.

La implementación de la telecolaboración supone una innovación en cuanto al modo en que se aprenden las lenguas, ya que el aprendizaje se produce más allá del aula (Dooly, 2010, 2012), en contextos reales de uso y con hablantes tanto nativos (O’Dowd y Ritter, 2006; Hauck y Youngs, 2008; Sevilla Pavón y Nicolaou, 2017) como no nativos (Guth y Helm, 2010; Kohn, 2011; Sevilla Pavón, 2018). De este modo, los estudiantes tienen la oportunidad de tener un contacto directo con la lengua y la cultura meta, o con otras culturas con una lengua meta común, gracias a las posibilidades que ofrecen los entornos virtuales de aprendizaje de corte colaborativo para innovar y enriquecer las clases presenciales.

\section{TELECOLABORACIÓN Y DESARROLLO DE COMPETENCIAS}

La telecolaboración o intercambio intercultural en línea (Online Intercultural Exchange, OIE) permite a los participantes en los intercambios explorar otras culturas y comunicarse con otros participantes en diferentes lugares del mundo, en un entorno virtual, apoyando la movilidad virtual del alumnado, el intercambio lingüístico, el intercambio intercultural y el aprendizaje de lenguas. Se plantea, pues, como una respuesta efectiva ante la anteriormente señalada falta de experiencias internacionales de una importante parte del alumnado, ya que reúne a estudiantes de diversos países en un mismo entorno virtual, lo cual es posible gracias al uso de las TIC. En dicho entorno, a través de la realización de tareas colaborativas por parte de los estudiantes, se fomenta un mejor entendimiento, compromiso y un aprendizaje por exploración y descubrimiento. Esta forma de aprender está orientada al fomento de competencias lingüísticas, interculturales, literarias y digitales. Así, los estudiantes 
practican la lengua meta o L2, ya sea mediante intercambios con hablantes nativos o, en el caso de la experiencia que nos ocupa, utilizando la L2 (el inglés) como "lengua franca" o lengua común entre los alumnos de diferentes países. Del mismo modo, los estudiantes adquieren progresivamente un conocimiento profundo y de primera mano de otras culturas, otras realidades e incluso de sí mismos, al tiempo que se familiarizan con las distintas herramientas TIC empleadas.

En los últimos años se han venido realizando diversos proyectos de telecolaboración a nivel internacional entre universidades de todo el mundo: CULTURA (Furstenbergy Levet, 2010), TeCOLA (Jauregi y Melchor-Couto, 2017), INTENT y UNI-collaboration (O’Dowd, 2015), y TILA (Kohn, 2016), entre otros. La presente propuesta gira en torno al proyecto iTECLA (Sevilla Pavón, 2018), en que se incorpora la creación de materiales y objetos de aprendizaje por parte de los propios estudiantes, así como el desarrollo de competencias que contribuyan a un cambio de enfoque en la educación superior a través de una metodología innovadora. Dicha metodología combina el intercambio intercultural en línea con la redacción e intercambio de cuentos del mundo.

Uno de los pilares y razones de ser de la telecolaboración es el fomento de la competencia intercultural. La importancia del desarrollo de la competencia intercultural en los estudiantes en nuestros días ha sido señalada en numerosas publicaciones (Byram, 2000, 1997; Bandura, 2003). Ello se ha relacionado frecuentemente con la telecolaboración en el aula y más allá de ella (Belz, 2003, 2007; O’Dowd y Waire, 2009; Vinagre Laranjeira, 2008, 2010b; Dooly y Sadler, 2013), debido a las múltiples posibilidades que esta modalidad de aprendizaje ofrece. De este modo, se incrementan los beneficios que para los participantes tienen los intercambios telecolaborativos en relación a aspectos tan importantes como diversos: la autonomía de los estudiantes (Hughes, 2010; Fuchs, Hauck y Müller-Hartmann, 2012), la fluidez y la precisión lingüística (Kinginger y Belz, 2005; O'Rourke, 2007; Guth y Helm, 2010), la competencia intercultural (MüllerHartmann, 2000; Ware, 2005; O'Dowd, 2006, 2010, 2003; Sevilla-Pavón, 2018), las destrezas de comunicación intercultural en línea (Belz y Müller-Hartmann, 2003; O'Dowd y Ritter, 2006; Guth y Helm, 2010), la competencia digital multimodal y las nuevas competencias online (Hauck, 2007; Guth y Helm, 2010), la pertenencia y participación activa en "comunidades de práctica" (Dooly y Sadler, 2013), la negociación de significados (Cantó, de Graaff y Jauregi Ondarra, 2014), la motivación de los estudiantes (Cantó, Jauregi Ondarra y van den Bergh, 2013), la formación de profesores de idiomas y el aprendizaje entre pares (Brígido Corachán, 2008; Guth y Helm, 2010; Kohn y Warth, 2011; Lewis, Chanier y Youngs, 2011; Cunningham, 2013; Dooly y Sadler, 2013), el pensamiento crítico y las destrezas de análisis (Furstenberg y Levet, 2010); entre otros.

El fomento de la competencia lingüístico-comunicativa intercultural está íntimamente relacionado con el enfoque de Educación para el Desarrollo (ED). Así, las tareas del proyecto incluyen temáticas en torno a los Objetivos de desarrollo 
sostenible (ODS) u Objetivos mundiales (UNESCO, 2015, 2017) recogidos en la Agenda 2030 para el desarrollo sostenible. Entre dichos ODS se incluyen 169 iniciativas en forma de 17 objetivos que tienen que ver con la erradicación de la pobreza, el cuidado del planeta, la eliminación de las desigualdades y la cooperación y compromiso entre todos los países de la ONU para alcanzar dichos objetivos. La siguiente figura muestra los 17 ODS que la UNESCO se propone alcanzar antes de 2030:

Figura 1. Los 17 Objetivos de desarrollo sostenible propuestos por la UNESCO (2016)

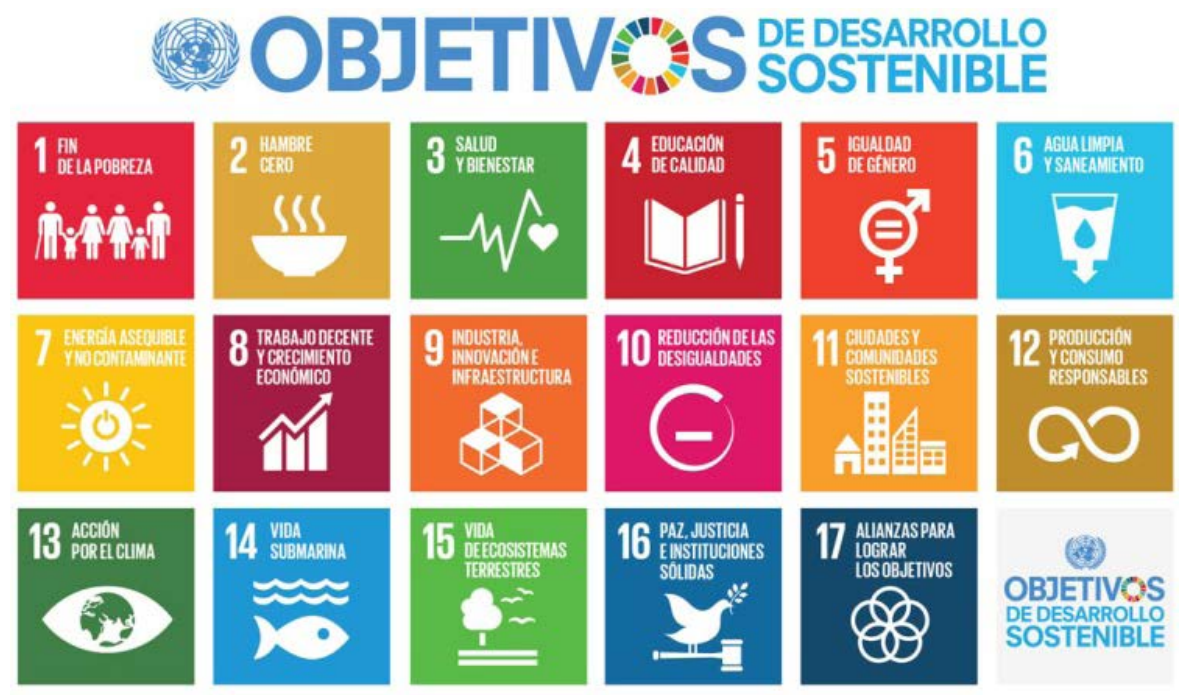

\section{ANTECEDENTES DEL PROYECTO}

Este proyecto se engloba en el marco de una iniciativa conjunta, fruto de la colaboración entre los grupos de investigación TALIS y SILVA a través del proyecto iTECLA. TALIS se ocupa de la producción y utilización de cuentos multilingües e interculturales para crear un mundo más respetuoso con los derechos humanos y con una mayor aceptación de la interculturalidad; al tiempo que establece una red formada por los participantes en las diferentes experiencias llevadas a cabo en torno a los cuentos del mundo y el desarrollo de competencias interculturales y lingüísticas (Alcantud Díaz, 2012; Alcantud Díaz, Martínez Usarralde y Lloret Català, 2017). La red de TALIS se estableció inicialmente con profesores y estudiantes de inglés y de español como lengua extranjera en la Universitat de València (España); y en la Queen Mary University (Reino Unido); añadiéndose posteriormente la Universidad de Krosno (Polonia) y la National and Kapodistrian University of Athens (Grecia). A 
estas instituciones les han seguido muchas otras en todo el mundo, entre las que se incluyen Cyprus University of Technology (CUT, Chipre) y Bulacan State University (BulSU, Filipinas).

Desde el inicio del proyecto, se han venido realizando infinidad de intercambios de telecolaboración entre más de mil estudiantes de diversos países y nacionalidades a través de herramientas de videoconferencia como Google Hangouts para la mejora de sus competencias lingüísticas, así como el desarrollo de sus competencias interculturales, literarias y digitales. La vigencia y relevancia del proyecto se refleja en el hecho de que la red de colaboración continúa creciendo, tanto en número de países como de idiomas, con participantes de orígenes muy diversos (Alcantud Díaz, López y Palomero, 2014).

\section{TAREAS DEL PROYECTO}

Como paso previo a la realización de las sesiones de telecolaboración y para garantizar que dichas sesiones se llevasen a cabo de manera efectiva y eficiente, se realizaron las acciones que se detallan a continuación:

\section{Elección de la modalidad de intercambio:}

La modalidad elegida fue intercambio de "lengua franca" (entre hablantes no nativos de la lengua estudiada por ambos grupos de estudiantes, siendo el inglés la lengua común entre ambos grupos). Así, los estudiantes de los dos países participantes eran hablantes no nativos del inglés que utilizaban dicha lengua para comunicarse entre sí.

\section{Elección de la institución colaboradora para llevar a cabo las sesiones:}

Para la elección de la institución, se optó por establecer la colaboración con una institución mediante el uso de la plataforma Unicollaboration.

\section{Elección del modo de comunicación: síncrono o asíncrono:}

Otra de las decisiones tenía que ver con determinar si las tareas se realizarían de manera síncrona o asíncrona. Se optó por una combinación de ambas, siempre teniendo en cuenta que, para emplear herramientas de comunicación síncrona (con el programa de videoconferencia Google Hangouts), era conveniente que no hubiera grandes diferencias horarias entre las instituciones participantes ya que, de lo contrario, se habrían de establecer todas las sesiones en línea fuera del horario de clase. Puesto que el intercambio tuvo lugar entre España y Chipre, la diferencia horaria era de tan solo una hora. 


\section{Establecimiento de principios comunes entre las instituciones colaboradoras:}

Se concertaron reuniones virtuales entre los docentes responsables del intercambio en ambas instituciones a través de Google Hangouts para discutir expectativas, objetivos y marcos temporales. También se compararon las guías docentes de las asignaturas y cursos en que se llevaría a cabo la experiencia. Asimismo, se escogieron cuentos que tratasen temas apropiados y relevantes para ambos grupos teniendo en cuenta que los temas habían de ser, además, interesantes desde el punto de vista intercultural e incluir diferentes ODS. Además, se especificó que el intercambio se basaría en la modalidad de "lengua franca”. Igualmente, se discutieron las herramientas a utilizar de entre las que ofrece la plataforma Google+.

\section{Diseño de las tareas:}

Se especificaron, en primer lugar, los objetivos de aprendizaje lingüísticos y comunicativos, interculturales, literarios y digitales. Por su parte, la tarea principal se planteó de tal manera que se pudiese desarrollar colaborativamente, fomentando la interdependencia positiva entre los participantes.

Puesto que los estudiantes no se conocían previamente, la primera actividad fue de precalentamiento y sirvió para romper el hielo y para que los alumnos se conociesen mutuamente. Seguidamente, se especificaron las tareas preparatorias y cada docente adaptó dichas tareas a su contexto específico para así garantizar una buena preparación de los estudiantes de cara a la telecolaboración. Asimismo, se especificó la secuenciación de tareas de telecolaboración.

Además, tras cada sesión, se realizó una post-tarea para garantizar resultados de aprendizaje, en forma de informe final, diario de reflexión/aprendizaje, actividad oral o escrita en clase o para casa, etc.

En cuanto a la preparación de los estudiantes, los docentes se encargaron de los siguientes aspectos: 
Tabla 1. Pasos previos a la realización de las tareas de telecolaboración

\begin{tabular}{|c|c|c|}
\hline Pasos & \multicolumn{4}{|c|}{ Actividades } \\
\hline $\mathbf{1}$ & $\begin{array}{l}\text { Preparación de los estudiantes para las tareas de telecolaboración, } \\
\text { principalmente en cuanto a la temática del cuento y a los medios de } \\
\text { expresión empleados, usando las tareas y actividades diseñadas. }\end{array}$ \\
\hline $\mathbf{2}$ & $\begin{array}{l}\text { Preparación de los estudiantes para la experiencia de intercambio } \\
\text { colaborativo, fomentando la apertura de mente y la empatía como } \\
\text { elementos facilitadores que permiten que aprendan los unos de los } \\
\text { otros y se ayuden mutuamente. }\end{array}$ \\
\hline $\mathbf{3}$ & $\begin{array}{l}\text { Familiarización de los alumnos con las herramientas de } \\
\text { telecolaboración. }\end{array}$ \\
\hline $\mathbf{5}$ & $\begin{array}{l}\text { Organización de los estudiantes en función de su nivel de } \\
\text { familiarización con los recursos tecnológicos empleados y para que } \\
\text { puedan ayudar a sus compañeros. }\end{array}$ \\
\hline $\begin{array}{l}\text { Provisión de instrucciones concretas para las tareas de } \\
\text { telecolaboración, repartiendo fichas de trabajo o explicitando los pasos } \\
\text { seguir en el Entorno virtual de aprendizaje (EVA) empleado en cada } \\
\text { institución. }\end{array}$ \\
$\begin{array}{l}\text { En los intercambios de comunicación síncrona a través de Google } \\
\text { Hangouts, comprobación del correcto funcionamiento de las diferentes } \\
\text { herramientas antes de comenzar la sesión en línea y diseño de un plan } \\
\text { B al que poder recurrir en caso de que la tecnología fallase. }\end{array}$ \\
\hline
\end{tabular}

Por su parte, entre las funciones de los docentes durante la realización de las sesiones de telecolaboración destacó el apoyo a sus estudiantes ante posibles dificultades de tipo técnico o lingüístico. Finalmente, entre las tareas posteriores a la sesión de telecolaboración destacaron:

Tabla 2. Pasos posteriores a la realización de las tareas de telecolaboración

\begin{tabular}{|c|c|c|}
\hline Pasos & Actividades \\
\hline $\mathbf{1}$ & $\begin{array}{l}\text { Provisión a los alumnos de tareas subsecuentes para garantizar el } \\
\text { aprendizaje significativo (en especial, después de realizar los } \\
\text { intercambios síncronos). }\end{array}$ \\
\hline $\mathbf{2}$ & $\begin{array}{l}\text { Elaboración de diarios de reflexión/aprendizaje por parte de los } \\
\text { alumnos para recabar opiniones y sugerencias de mejora. }\end{array}$ \\
\hline $\mathbf{3}$ & $\begin{array}{l}\text { Retroalimentación en cuanto a los problemas o logros de tipo } \\
\text { lingüístico, literario, comunicativo, intercultural o técnico. }\end{array}$ \\
\hline
\end{tabular}

En cuanto al diseño de las tareas, cada docente se aseguró de tener en cuenta la integración pedagógica en el currículum. Para ello, las tareas no se diseñaron como unidades aisladas sino como secuencias más complejas de sub-tareas que incluyeron tareas preparatorias, tareas principales y tareas subsiguientes. 
Asimismo, la especificación de la telecolaboración se llevó a cabo teniendo en cuenta que, tal y como señalamos anteriormente, las tareas suelen basarse en la combinación de secuencias de sub-tareas de aprendizaje organizadas en 3 fases: preparatoria, principal y posterior.

\section{OBJETIVOS}

El objetivo principal del proyecto es el fomento de la telecolaboración entre estudiantes de diversas culturas y países de origen para el desarrollo de competencias lingüísticas, interculturales, literarias y digitales a través de la realización de tareasen las modalidades de telecolaboración síncrona y asíncrona- basadas en los cuentos del mundo.

Por su parte, el objetivo secundario que el proyecto persigue tiene que ver con la ampliación de la red de telecolaboración formada por los participantes en las diferentes experiencias llevadas a cabo por TALIS en torno a la creación e intercambio de cuentos del mundo.

\section{METODOLOGÍA}

\section{Contexto y cuestiones preliminares}

Para alcanzar los objetivos, fue necesario llevar a cabo una serie de acciones que tienen que ver con decisiones en cuanto al diseño de tareas y en relación a los materiales empleados. En primer lugar, se adaptaron una serie de materiales pedagógicos ya existentes en torno a los cuentos del mundo, de tal manera que pudieran ser utilizados en las tareas de telecolaboración propuestas. Seguidamente, se pusieron en marcha los intercambios de telecolaboración entre las instituciones participantes, produciéndose intercambios de tipo L2 o "lengua franca", como se explicaba anteriormente.

Estos intercambios se llevaron a cabo durante dos horas semanales a lo largo de 10 semanas, durante el primer semestre del curso académico 2016-2017. Para evitar posibles inconvenientes derivados de la diferencia horaria, de la limitada disponibilidad de estudiantes y profesores y de las dificultades de acceso simultáneo a los recursos TIC necesarios, se priorizaron los intercambios de tipo asíncrono, con lo que cada una de las partes participantes podía realizar las diferentes tareas en el horario más conveniente. Determinadas tareas se realizaron en grupos pequeños (de un máximo de 4 alumnos), mientras que otras tareas se realizaron por parejas, siempre respetando las posibles combinaciones anteriormente mencionadas y teniendo en cuenta a la hora de hacer las parejas y equipos, además, la afinidad de los estudiantes participantes en cuanto a nivel, gustos y aficiones, grado estudiado y edad. 


\section{Participantes, instrumentos y materiales}

Los participantes en el estudio piloto aquí descrito fueron 14 estudiantes $(\mathrm{N}=14)$ de diferentes países: chipriotas $(n=8)$, keniatas $(n=3)$ y nigerianos $(n=1)$, todos ellos estudiantes de la Cyprus University of Technology (CUT), Chipre, quienes realizaron una serie de actividades fruto de un convenio entre la CUT y la Universitat de València (UV), España, el cual incluía un intercambio intercultural en línea que se ha venido llevando a cabo durante los 3 últimos años. Sus edades estaban comprendidas entre los 18 y los 25 años, y se encontraban estudiando diversos grados de los ámbitos científicos, sociales y sanitarios: diversos grados en ingeniería, enfermería y administración de empresas, entre otros.

En el marco de este intercambio, se realizó un taller de escritura e ilustración creativas a través del cual se obtuvieron los datos analizados en este artículo, mediante un cuestionario final que obtuvo 10 respuestas, tanto cuantitativas como cualitativas, por parte de los participantes que completaron todas las tareas del proyecto. La parte cuantitativa de este cuestionario presentaba, en primer lugar, una sección inicial de datos demográficos (edad, estudios realizados, lugar de procedencia). La segunda sección consistía en 5 preguntas de respuesta cerrada, de una escala Likert de 7 puntos (siendo 1 "muy en desacuerdo" y 7 "muy de acuerdo"), en relación a afirmaciones sobre diversos aspectos del proyecto: cumplimiento de las expectativas de los participantes; consecución de los objetivos; tareas y contenidos, y organización de los mismos; nivel de interés suscitado por el proyecto; nivel de aplicabilidad en su actividad profesional presente o futura; duración; y metodología didáctica. Por último, la tercera sección del cuestionario se centraba en la respuestas abiertas de corte cualitativo, en que los participantes completaban enunciados sobre: cómo definirían el proyecto en una sola palabra o frase breve; qué habían aprendido; qué les había gustado más y menos; qué añadirían o quitarían; y qué sugerirían para mejorar el proyecto. Estos datos se complementaron con los comentarios y opiniones de los participantes, recogidos mediante observación en el aula y diarios de campo.

Los principales materiales empleados para llevar a cabo las tareas fueron diferentes volúmenes de los cuentos del mundo resultantes de ediciones anteriores del proyecto (un volumen por cada pareja o pequeño grupo de estudiantes), los ordenadores de una sala de informática y el programa gratuito de videoconferencia Google Hangouts.

\section{RESULTADOS Y DISCUSIÓN}

El análisis de los diferentes datos recabados muestra, en primer lugar, que las expectativas de los participantes en relación al proyecto se cumplieron en el 90\% de los casos, ya que solo uno de los participantes manifestó que el proyecto no había cumplido completamente sus expectativas. Además, los participantes otorgaron las puntuaciones máximas en relación a sus percepciones del desarrollo 
de la creatividad, obteniendo este ítem una puntuación de 5,8 (en una escala Likert de 7 puntos). También el resto de aspectos estudiados obtuvieron puntuaciones satisfactorias: desarrollo de competencias literarias (obteniendo una puntuación de 5,2 ), lingüísticas (con una valoración de 5,7), interculturales (valorado con 5,2) y digitales (puntuadas con un 5,6), tal y como se refleja a continuación:

Figura 2. Valoración de los participantes en el proyecto en cuanto al desarrollo de competencias

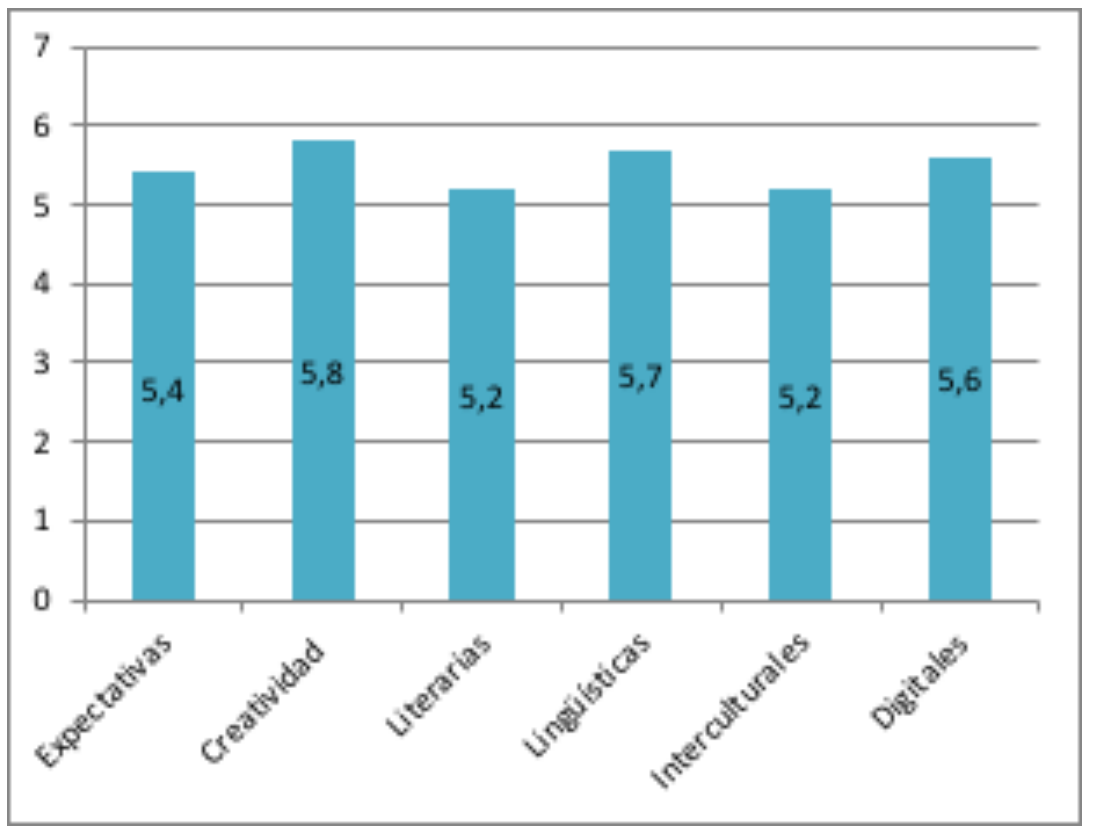

Las respuestas cualitativas a los enunciados propuestos complementaron las percepciones y opiniones expresadas por los estudiantes de manera numérica. En primer lugar, se les pidió que definieran el proyecto de manera breve. En sus respuestas, los participantes destacaron aspectos de tipo cultural, como muestran las respuestas 1 a 3 de la tabla 3 . 
Tabla 3. Valoraciones globales del proyecto

\begin{tabular}{|l|l|}
\hline $\begin{array}{l}\text { Respuesta 1: "a good project which enables } \\
\text { people to know various stuff from different } \\
\text { communities all over the world" }\end{array}$ & $\begin{array}{l}\text { (un buen proyecto que permite } \\
\text { conocer varios aspectos de } \\
\text { derentes comunidades de todo el } \\
\text { mundo) }\end{array}$ \\
\hline $\begin{array}{l}\text { R2: "It's a place where we learn to appreciate } \\
\text { the world through culture" }\end{array}$ & $\begin{array}{l}\text { (es un lugar donde aprendemos a } \\
\text { apreciar el mundo a través de la } \\
\text { cultura) }\end{array}$ \\
\hline $\begin{array}{l}\text { R3: "a group of people engage in intensive } \\
\text { discussion and activity on a particular subject" }\end{array}$ & $\begin{array}{l}\text { (un grupo de personas mantienen un } \\
\text { debate intenso y una actividad en } \\
\text { relación a un tema concreto) }\end{array}$ \\
\hline
\end{tabular}

Además, los participantes completaron el enunciado respecto a lo aprendido con respuestas que muestran que valoraron especialmente la oportunidad de trabajar en equipo y de aprender sobre diferentes culturas, como puede apreciarse en las respuestas 4 a 6 de la tabla 4 .

Tabla 4. Aspectos más destacados del proceso de aprendizaje

\begin{tabular}{|l|l|}
\hline $\begin{array}{l}\text { R4: "what other communities in the world did or } \\
\text { believed long ago or until now" }\end{array}$ & $\begin{array}{l}\text { lo que otras comunidades en el } \\
\text { mundo hicieron o creyeron hace } \\
\text { mucho tiempo o hasta la actualidad) }\end{array}$ \\
\hline R5: "cooperation" & \begin{tabular}{l} 
(cooperación) \\
\hline $\begin{array}{l}\text { R6: "working like a team and writing based on } \\
\text { our ideas" }\end{array}$
\end{tabular} $\begin{array}{l}\text { (el trabajo en grupo y la escritura } \\
\text { basada en nuestras ideas) }\end{array}$ \\
\hline
\end{tabular}

En cuanto a los aspectos que más y que menos les habían gustado del proyecto, los participantes valoraron positivamente los aspectos lingüísticos e interculturales del mismo, la posibilidad de compartir aspectos de su cultura, y el carácter lúdico de la propuesta, como se aprecia en sus respuestas. Por su parte, la restricción temporal a la hora de llevar a cabo las actividades del proyecto fue el aspecto peor valorado del proyecto. Las siguientes afirmaciones, recogidas en las respuestas 7 a 10 de la tabla 5 , muestran los aspectos positivos y negativos señalados por los estudiantes. 
Tabla 5. Aspectos positivos y negativos del proyecto

\begin{tabular}{|l|l|}
\hline $\begin{array}{l}\text { R7: "II liked the fact that] it promotes linguistics, } \\
\text { culture and learning about myths" }\end{array}$ & $\begin{array}{l}\text { (me gustó el hecho de que) } \\
\text { fomenta la lingüística, la cultural y el } \\
\text { aprendizaje sobre mitos }]\end{array}$ \\
\hline R8: "[I liked] the playful nature of the project"” & $\begin{array}{l}\text { (me gustó) el carácter lúdico del } \\
\text { proyecto] }\end{array}$ \\
\hline R9: "[I did not like] not having enough time" & $\begin{array}{l}\text { (no me gustó) no tener tiempo } \\
\text { suficiente] }\end{array}$ \\
\hline
\end{tabular}

El último enunciado en la sección de preguntas de respuesta abierta permitió a los participantes aportar sugerencias de mejora en cuanto al proyecto. Entre dichas sugerencias, destacaron el interés por tener acceso a un mayor número de cuentos y a una mayor variedad de culturas de todo el mundo; más aprendizaje sobre la historia de los diferentes países implicados; más tiempo disponible para completar las tareas; y más recursos visuales, como reflejan las siguientes respuestas de la tabla 6:

Tabla 6. Sugerencias de mejora del proyecto

\begin{tabular}{|l|l|}
\hline R11: "More history" & (más historia) \\
\hline R12: "More visuals" & (más recursos visuales) \\
\hline $\begin{array}{l}\text { R13: "More tales and cultures from different } \\
\text { parts of the world" }\end{array}$ & $\begin{array}{l}\text { (más cuentos y culturas de } \\
\text { diferentes lugares del mundo) }\end{array}$ \\
\hline R14: "A little more time" & (un poco más de tiempo) \\
\hline
\end{tabular}

En lo que respecta al trabajo realizado por los participantes, el número total de cuentos creados por los mismos fue de 7 cuentos procedentes de 3 países (Chipre, Kenia y Nigeria), como muestra la siguiente tabla: 
Tabla 7. Cuentos resultantes de la iniciativa de Cuentos de Chipre

\begin{tabular}{|c|c|c|}
\hline Cuento y autor/a/os/as & País & Lenguas \\
\hline The Tiger (Ade Sco) & Nigeria & $\begin{array}{c}\text { Yoruba, griego chipriota, } \\
\text { inglés }\end{array}$ \\
\hline The Prophecy (Brian Kibet) & Kenia & $\begin{array}{c}\text { Nandi- Markweta } \\
\text { (Kalenjin), griego } \\
\text { chipriota, inglés }\end{array}$ \\
\hline Avalon (Elena Ppeli y Spiros Efrem) & Chipre & $\begin{array}{c}\text { Griego chipriota, español, } \\
\text { inglés }\end{array}$ \\
\hline $\begin{array}{c}\text { The Gentle Giant (Antonis } \\
\text { Charalambous, Stavros Nikola y Anna- } \\
\text { Maria Evagorou) }\end{array}$ & Chipre & $\begin{array}{c}\text { Griego chipriota, español, } \\
\text { inglés }\end{array}$ \\
\hline $\begin{array}{c}\text { The Legend of Saint George (Lefkios } \\
\text { Christodoulou y Christos Hadjipanavi) }\end{array}$ & Chipre & $\begin{array}{c}\text { Griego chipriota, español, } \\
\text { inglés }\end{array}$ \\
\hline The Goblin (Panagiota Vasiliou) & Chipre & $\begin{array}{c}\text { Griego chipriota, español, } \\
\text { inglés }\end{array}$ \\
\hline
\end{tabular}

Los temas tratados en los cuentos están íntimamente relacionados con los ODS y con la Educación para el Desarrollo (Sevilla-Pavón y Nicolaou, 2017; UNESCO, 2015), como puede apreciarse en la siguiente tabla:

Tabla 8. Presencia de los ODS en los Cuentos de Chipre

\begin{tabular}{|c|c|c|c|c|c|c|}
\hline Objetivos de desarrollo sostenible & $\begin{array}{l}\text { The } \\
\text { Tiger }\end{array}$ & The Prophecy & Avalon & $\begin{array}{c}\text { The } \\
\text { Gentle } \\
\text { Giant }\end{array}$ & $\begin{array}{c}\text { The } \\
\text { Legend of } \\
\text { Saint } \\
\text { George } \\
\end{array}$ & $\begin{array}{l}\text { The } \\
\text { Goblin }\end{array}$ \\
\hline $\begin{array}{l}1 \text { y } 2 \text { - Erradicación de la pobreza y } \\
\text { del hambre }\end{array}$ & & $\mathrm{x}$ & $\mathrm{x}$ & & & \\
\hline 3- Salud y bienestar & $\mathrm{x}$ & & & & $\mathrm{x}$ & \\
\hline 6- Agua limpia y saneamiento & & & & & $\mathrm{x}$ & \\
\hline 8- Empleo y crecimiento económico & & $\mathrm{x}$ & & & & \\
\hline 10- Reducción de las desigualdades & & $\mathrm{x}$ & $\mathrm{x}$ & $\mathrm{x}$ & & $\mathrm{x}$ \\
\hline 13- Acción por el clima y el entorno & & $\mathrm{x}$ & & $\mathrm{x}$ & & \\
\hline 14- Vida acuática & $\mathrm{x}$ & & & & & \\
\hline 15- Vida terrestre & $\mathrm{x}$ & $\mathrm{x}$ & & $\mathrm{x}$ & & $x$ \\
\hline 16- Paz y justicia & $\mathrm{x}$ & $\mathrm{x}$ & $\mathrm{x}$ & $\mathrm{x}$ & $\mathrm{x}$ & \\
\hline 17- Alianzas internacionales & & $\mathrm{x}$ & $\mathrm{x}$ & & & \\
\hline
\end{tabular}


Tal y como muestra la tabla anterior, un total de once ODS se incluyeron en los cuentos. De entre ellos, los ODS con mayor presencia fueron: "10- Reducción de las desigualdades" y "16- Paz y justicia", presentes en cuatro y cinco de los cuentos respectivamente; "15-Vida terrestre", que aparece en cuatro de los cuentos; "1 y 2- Erradicación de la pobreza y del hambre" , "17- Alianzas internacionales", que aparecen en un cuento; "13- Acción por el clima y el entorno" y "3-Salud y bienestar", que figuran en dos de los cuentos cada uno. Además, también aparecen en al menos uno de los cuentos los siguientes ODS: "14- Vida acuática", "8- Empleo y crecimiento económico", “17- Alianzas internacionales” y "6- Agua limpia y saneamiento”.

\section{CONSIDERACIONES FINALES}

Este artículo describe una iniciativa conjunta de los grupos TALIS y SILVA y del proyecto iTECLA en torno al proceso de diseño de intercambios de telecolaboración entre instituciones de diferentes países. Dicho proceso, ya sea en su modalidad de tándem de Lengua materna L1 o de "lengua franca" L2, se caracteriza por su gran complejidad. Es necesaria una considerable inversión de tiempo en el diseño de las tareas de los intercambios para garantizar que estos se lleven a cabo de manera exitosa y con el menor número posible de imprevistos. De este modo, se favorece la consecución de los objetivos de aprendizaje esperados, a nivel lingüístico, literario, digital e intercultural, para beneficio de los estudiantes participantes en el proyecto.

Los intercambios de tipo asíncrono ofrecen la gran ventaja de que los alumnos realizan las tareas en sus propios tiempos y horarios, y así se tratan de evitar inconvenientes derivados de la diferencia horaria, de la limitada disponibilidad horaria de estudiantes y profesor o de las restricciones de acceso a los recursos TIC necesarios. A pesar de ello y de su gran complejidad, los intercambios síncronos también son de gran utilidad por proporcionar una experiencia de comunicación en vivo y en directo que contribuye a crear una mayor cercanía entre los hablantes, favoreciendo el entendimiento intercultural. Además, la comunicación en tiempo real hace que los intercambios sean más directos y personales, lo cual fortalece el nivel de compromiso de los estudiantes para con las tareas y resulta, de manera general, motivador para los participantes.

Uno de los factores clave para garantizar el éxito de la telecolaboración es que el docente haga una preparación adecuada de los estudiantes, previa a las sesiones de intercambio. Así, el docente ha de: introducir el tema de la telecolaboración y los medios de expresión; fomentar una actitud abierta, empática y colaborativa; asegurarse de que los alumnos estén familiarizados con las herramientas que se usarán; fomentar el aprendizaje entre pares para que los alumnos más expertos ayuden a los menos familiarizados con la tecnología; proporcionar instrucciones claras en relación a las tareas a realizar; y comprobar el correcto funcionamiento de las herramientas que se vayan a emplear. Todo ello favorece el óptimo desempeño en las sesiones de telecolaboración y permite al docente estar más tranquilo y seguro 
durante su ejecución, lo cual posibilita el correcto ejercicio de su papel como facilitador y supervisor del proceso. Por último, el resto de factores que permiten asegurar que las sesiones de telecolaboración lleguen a buen puerto tienen que ver con el correcto diseño de las tareas, con sus correspondientes pre-tareas, tareas principales y posttareas, todas ellas con objetivos pedagógicos claros, y correctamente integradas en el currículum.

Este proyecto se centra en el fomento de la competencia digital, lingüística, intercultural y literaria a través de la realización telecolaborativa de tareas en torno a los cuentos del mundo. Se trata de un proyecto innovador, por permitir la construcción y adquisición del conocimiento más allá del espacio físico del aula de idiomas; y por atender una importante necesidad detectada en cuanto al aprendizaje de idiomas y el desarrollo de diversas competencias de la población universitaria europea ya que, actualmente, el 90\% de la misma no realiza parte de sus estudios o formación en el extranjero.

Los resultados del estudio piloto apuntan a la consecución de los objetivos y ponen de manifiesto el gran potencial pedagógico de las tareas telecolaborativas llevadas a cabo en contextos de aprendizaje de lenguas extranjeras. El proyecto logró aumentar la concienciación de los participantes en relación a los ODS, lo cual quedó reflejado en el alto número y gran variedad de ODS presentes en los diferentes cuentos. Además, de acuerdo con las opiniones vertidas por los estudiantes, el proyecto de telecolaboración a través de cuentos del mundo favoreció el desarrollo de su creatividad, así como la competencia intercultural, lingüística, literaria y digital. Una vez concluido, los participantes consideraron de manera mayoritaria que sus expectativas en relación al proyecto se habían satisfecho.

En cuanto a los aspectos mejor valorados del proyecto, de acuerdo con las afirmaciones de los estudiantes, estos fueron: el conocimiento e intercambio con otras culturas, el trabajo colaborativo en equipo, el fomento de competencias lingüísticas e interculturales, y el carácter lúdico de la propuesta.

A pesar de destacar un gran número de aspectos positivos en relación al proyecto, los participantes también manifestaron sus críticas, la inmensa mayoría de las cuales se dirigieron al tiempo disponible, que consideraron escaso. Asimismo, los participantes aportaron sugerencias de mejora que se espera puedan enriquecer las ediciones futuras del proyecto.

Por tratarse de un estudio piloto con una muestra de tamaño reducido, los resultados deben interpretarse con cautela. Del mismo modo, el hecho de que los datos obtenidos se basen en las percepciones de los estudiantes supone una limitación del estudio. Los estudios futuros deberían realizarse con una muestra mayor. Además, para la obtención de resultados más concluyentes, sería recomendable que los estudios futuros mostraran una comparación entre a) las percepciones de los estudiantes en cuanto al desarrollo de competencias y b) sus resultados reales en pruebas objetivas. 


\section{REFERENCIAS BIBLIOGRÁFICAS}

Alcantud Díaz, M., Martínez Usarralde, M. J., y Lloret Català, M. C. (2017). Service-Learning and Project TALIS. Pedagogy and Teaching destined to mutual understanding. Pedaqogika, 126(2), 228-247. doi: https://doi. org/10.15823/p.2017.31

Alcantud Díaz, M., López, I., y Palomero, R. (Coords.) (2014). Cuentos alrededor del Mundo - Stories around the World, Vol. 1 y 2. Madrid: Vaughan Systems.

Alcantud Díaz, M. (Coord.) (2012). Cuentos del Mundo - Tales of the World, vol. 1-6. Madrid: DualBooks.

Altbach, P. G., y Knight, J. (2007). The internationalization of higher education: Motivations and realities. Journal of Studies in International Education, 11(3), 290-305.

Bandura, A. (2003). The concept of intercultural competence. Interview with Prof. M. Byram, Symposium on Intercultural Competence and Education for Citizenship, School of Education, University of Durham, UK.

Belz, J. (2007). The development of intercultural communicative competence. In R. O’Dowd (Ed.), On-line Intercultural Exchange: An Introduction for Foreign Language Teachers. Clevedon: Multilingual Matters, 127-166.

Belz, J. (2003). Linguistic perspectives on the development of intercultural competence in telecollaboration. Language Learning and Technology, 7(2), 68-117.

Belz, J. A., y Müller-Hartmann, A. (2003). Teachers as intercultural learners: Negotiating German-American telecollaboration along the institutional fault line. The Modern Language Journal, 87(1), 71-89.

Brígido Corachán, A. M. (2008). Collaborative e-learning in the European Higher Education Area (EHEA): Towards a peer-assisted construction of knowledge.
GRETA. Revista para Profesores de Inglés, 16, 14-18.

Byram, M. (2000). Assessing intercultural competence in language teaching. Sprogforum, 18(6), 8-13.

Cantó, S., de Graaff, H. C. J., y Jauregi Ondarra, M. K. (2014). Collaborative tasks for negotiation of intercultural meaning in virtual worlds and video-web communication. En M. González Lloret y L. Ortega (Eds.), Technology-mediated TBLT: Researching technology and tasks. Amsterdam: Benjamins, 183-211.

Byram, M. (1997). Teaching and Assessing Intercultural Communicative Competence. Clevedon: Multilingual Matters.

Cantó, S., Jauregi Ondarra, M. K., y van den Bergh, H. H. (2013). Integrating cross-cultural interaction through videocommunication and virtual worlds in foreign language teaching programs. Burden or added value? ReCALL, 25(1), 105-121.

Castells, M. (2001). La galaxia Internet. Barcelona: Areté.

Chapelle, C. A., y Hegelheimer, V. (2004). The language teacher in the 21st century. En S. Fotos y C. M. Browne (Eds.), New Perspectives on CALL for Second Language Classrooms Mahwah. New Jersey: Lawrence Erlbaum Associates, 299-316.

Comisión Europea (2013). Number of Erasmus students tops 3 million. Recorte de prensa. Bruselas: Comisión Europea. Recuperado de http://europa.eu/rapid press-release IP-13-657 en.htm

Compton, L. K. L. (2009). Preparing language teachers to teach language online: a look at skills, roles, and responsibilities. Computer Assisted Language Learning, 22(1), 73-99.

Cunningham, D. J. (2013). Teacher competences in telecollaboration: The 
case of Web conferencing and German for professional purposes. In J. Aitken (Ed.), Cases on communication technology for second language acquisition and cultural learning, Hershey, PA: IGI Global, 173205.

Dooly, M., y Sadler, R. (2013). Filling in the gaps: Linking theory and practice through telecollaboration in teacher education. ReCALL, 25(1), 429.

Dooly, M. (2012). Divergent perceptions of telecollaborative language learning tasks: Task-as-workplan vs. task-as-process. Language Learning \& Technology, 15(2), 69-91.

Dooly, M. (2010). The teacher 2.o. In: Guth, S. and Helm, F. (eds.), Telecollaboration 2.0: Language, Literacies and Intercultural Learning in the 21st Century. Dooly, M. D. and O'Dowd, R. (series eds.), Telecollaboration in Education. Bern: Peter Lang, 277-303.

Ellis, R. (2000). Task-based research and language pedagogy. Language teaching research, 4(3), 193-220.

Fuchs, C., Hauck, M., y Müller-Hartmann, A. (2012) Promoting learner autonomy through multiliteracy skills development in cross-institutional exchanges. Language Learning \& Technology, 16(3), 82-102.

Furstenberg, G., y Levet, S. (2010). Integrating Telecollaboration into the Language Classroom: Some Insights. En M. Dooly y R. O’Dowd (Eds.), Telecollaboration 2.o for Language and Intercultural Learning. Bern: Peter Lang, 305-336.

Gee, J. P. (2007). What video games have to teach us about learning and literacy (Revised and Updated Edition). New York: Palgrave Macmillan.

Guth, S., y Helm, F. (Eds.) (2010). Telecollaboration 2.o: Language, Literacies, and Intercultural Learning in the 21st Century. Bern: Peter Lang.
Hauck, M. (2010). Telecollaboration: At the Interface between Multimodal and Intercultural Communicative Competence. En S. Guth y F. Helm (Eds.), Telecollaboration 2.o: Language, literacies and intercultural learning in the 21st century. Bern: Peter Lang, 219248.

Hauck, M. (2007). Critical success factors in a TRIDEM exchange. ReCALL, 19, 202223.

Hughes, J. (2010). The Multilingual Internet. In S. Guth y F. Helm (Eds.), Telecollaboration 2.0: Language, Literacies and Intercultural Learning in the 21st Century. Bern: Peter Lang.

Jauregi, K., y Melchor-Couto, S. (2017). The TeCoLa project: pedagogical differentiation through telecollaboration and gaming for intercultural and content integrated language teaching. En K. Borthwick, L. Bradley y S. Thouësny (Eds.), CALL in a Climate of Change: Adapting to Turbulent Global Conditions. Short Papers from EUROCALL 2017. Dublin: Research-publishing.net, 163169.

Kinginger, C., y Belz, J. A. (2005). Sociocultural perspectives on pragmatic development in foreign language learning: Case studies from telecollaboration and study abroad. Intercultural Pragmatics, 2, 369-421.

Kohn, K. (2016). From ELF communication to lingua franca pedagogy. En M. Pitzl y R. Osimk-Teasdale (Eds.), English as a Lingua Franca: Perspectives and Prospects: Contributions in Honour of Barbara Seidlhofer Boston/Berlin: De Gruyter Mouton, 87-96.

Kohn, K. (2011). English as a lingua franca and the Standard English misunderstanding. En A. De Houwer y A. Wilton (Eds.), English in Europe Today: Sociocultural and educational perspectives. AILA Applied Linguistics Series, 8 Amsterdam: John Benjamins Publishing, 71-94. 
Kohn, K., y Warth, C. (Eds.) (2011). Web collaboration for intercultural language learning. A guide for language teachers, teacher educators and student teachers. Münster: Monsenstein und Vannerdat.

Lewis, T., Chanier, T., y Youngs, B. (2011). Special Issue Commentary: Multilateral online exchanges for language and culture learning. Language Learning \& Technology, 15(1), 3-9.

Lichy, J., y Favre, B. (2018). Leaving the comfort zone: Building an international dimension in higher education. En J. J. Turner y G. Mulholland (Eds.), International Enterprise Education: Perspectives on Theory and Practice. London: Routledge.

Monereo, C., y Pozo, J. I. (2008). El alumno en entornos virtuales: condiciones, perfil y competencias. En C. Coll y C. Monereo (Eds.), Psicología de la Educación Virtual. Madrid: Morata.

Müller-Hartmann, A. (2000). The role of tasks in promoting intercultural learning in electronic learning networks. Language Learning and Technology, 4(2), 129-147.

Nunan, D. (1991). Communicative Tasks and the Language Curriculum. TESOL Quarterly, 25(2), 279-295.

O'Dowd, R. (2015). Supporting in-service language educators in learning to telecollaborate. Language Learning \& Technoloqu, 19(1), 64-83. Recuperado de http://llt.msu.edu/issues/february2015 odowd.pdf

O'Dowd, R. (2010). Issues in the assessment of online interaction and exchange. En S. Guth y F. Helm (Eds.), Telecollaboration 2.o: Language, literacies and intercultural learning in the 21st century Bern: Peter Lang, 337-360.

O'Dowd, R. (2006). Telecollaboration and the Development of Intercultural Communicative Competence. Munich: Langenscheidt-Longman.

O'Dowd, R. (2003). Understanding the 'other side': intercultural learning in a Spanish-
English e-mail exchange. Language Learning and Technology, $7(2), 118-144$.

O'Dowd, R., y Ritter, M. (2006). Understanding and working with 'failed communication' in telecollaborative exchanges. CALICO Journal, 23(3), 623642.

O'Dowd, R., y Waire, P. (2009). Critical issues in telecollaborative task design. Computer Assisted Language Learning, 22(2), 173-188.

O'Rourke, B. (2007). Models of telecollaboration (1): eTandem. In R. O'Dowd (Ed.), Online Intercultural Exchange Clevedon: Multilingual Matters, 41-61.

Piqueras Haba, J. (2011). El mundo en movimiento. Migración internacional y globalización. Cuadernos de Geografía, gO, 187-210. Recuperado de http:/ roderic.uv.es/handle/10550/39799

Sevilla-Pavón, A., (2018). L1 versus L2 online intercultural exchanges for the development of 21st century competences: the students' perspective. British Journal of Educational Technology, British Journal of Educational Technology, Early View, 1-27. doi: 10.1111/bjet.12602, Recuperado de http://onlinelibrary.wiley. com/doi/10.1111/bjet.12602/full

Sevilla-Pavón, A., (2017). La dimensión creativa en la escritura, ilustración y musicalización de cuentos del mundo. Revista Iberoamericana de Educación, 75(1), 89-110. Recuperado de https:/ rieoei.org/RIE/article/view/1357

Sevilla Pavón, A. (2015). Computer assisted language learning and the internationalisation of the Portuguese language in higher education contexts. Digital Education Review, 28, 37-44. Recuperado de http://revistes.ub.edu index.php/der/article/view/12033

Sevilla-Pavón, A., y Nicolaou, A. (2017) Online Intercultural Exchanges Through Digital Storytelling. International Journal of Computer-Assisted Language 
Learning and Teaching (IJCALLT), 7(4), 44-58. IGI Global. doi: 10.4018/ IJCALLT.2017100104.

SILVA Group. Recuperado de www.silvaunit. com

TALIS Project. Recuperado de www. proyectotalis.com

Tomlinson, B. (Ed.) (2011). Materials Development in Language Teaching. Cambridge: Cambridge University Press.

Teichler, U. (2004). The Changing Debate on Internationalisation of Higher Education. Higher Education, 48(1), 5-26.

UNESCO (2017). Education for Sustainable Development Goals: Learning Objectives. Paris: UNESCO. Recuperado de http://unesdoc.unesco.org/ mages/0024/002474/247444e.pdf

UNESCO (2016). Objetivos de desarrollo sostenible. Recuperado de http:/ www.un.org/sustainabledevelopment/ blog/2016/03/un-statisticalcommission-endorses-global-indicatorframework

UNESCO (2015). Transforming our World: The 2030 Agenda for Sustainable Development. United Nations Sustainable Development knowledge platform. General Assembly Distr.: General 21 October 2015, Seventieth session, Agenda items 15 and 116 Resolution adopted by the General Assembly on 25 September 2015. Recuperado de https://sustainabledevelopment.un.org/ post2015/transformingourworld/ publication

UNESCO (2008). Estándares de competencias en TIC para docentes (ECD-TIC). París: UNESCO.

UNESCO (1998). Informe mundial sobre la educación: Los docentes y la enseñanza en un mundo en mutación. Madrid: UNESCO/Santillana.

Vinagre Laranjeira, M. (2010a). Teoría y práctica del aprendizaje asistido por ordenador. Madrid: Síntesis.

Vinagre Laranjeira, M. (2010b). El aprendizaje intercultural en entornos virtuales de colaboración. RESLA, 23, 297-317.

Vinagre Laranjeira, M. (2008). Politeness strategies in collaborative e-mail exchanges. Computers \& Education, 5o, 1022-1036.

Ware, P. D. (2005). 'Missed' communication in online communication: Tensions in a German American telecollaboration. Language Learning \& Technology, 9(2), 64-89.

Willis, D., y Willis, J. (2007). Doing Task-based Teaching. Oxford: Oxford University Press.

\section{AGRADECIMIENTOS}

La autora de este artículo quisiera expresar su agradecimiento a la Conselleria de Educación de la Generalitat Valenciana su apoyo mediante la financiación del proyecto iTECLA: Entornos telecolaborativos innovadores de adquisición de lenguas para fines específicos (Ref. GV/2017/151, Programa Subvenciones para la realización de proyectos de $\mathrm{I}+\mathrm{D}+\mathrm{i}$ desarrollados por grupos de investigación emergentes, periodo 2017-2019). 


\section{PERFIL ACADÉMICO Y PROFESIONAL DE LA AUTORA}

Ana Sevilla-Pavón. Doctora en Lingüística Aplicada y profesora de inglés en la Universitat de València. Investigadora del Instituto IULMA y de los grupos SILVA y TALIS. Directora del proyecto iTECLA (GVA) y coordinadora del proyecto TALIS: Cuentos de Filipinas (Cátedra UNESCO/UV). Sus líneas de investigación incluyen: enseñanza y evaluación de lenguas asistidas por ordenador, inglés para fines específicos, formación docente en innovación educativa y comunicación intercultural a través de la telecolaboración.

E-mail: ana.m.sevilla@uv.es

\section{DIRECCIÓN DE LA AUTORA}

Departamento de Filología Inglesa y Alemana

Facultad de Filología, Traducción y Comunicación

Universitat de València

Avenida de Blasco Ibáñez, 32

46010 Valencia (España)

Fecha de recepción del artículo: 02/01/2018

Fecha de aceptación del artículo: 08/03/2018

\section{Como citar este artículo:}

Sevilla-Pavón, A. (2018). Diseño de tareas telecolaborativas para el aprendizaje de idiomas con cuentos del mundo. RIED. Revista Iberoamericana de Educación a Distancia, 21(2), pp.325-346. doi: http://dx.doi.org/10.5944/ried.21.2.20783 